\title{
FUNCTIONALS OF RATIONAL TYPE OVER THE CLASS $S$
}

\author{
LOUIS BRICKMAN
}

\begin{abstract}
Let $L$ be a continuous linear functional on the space of functions holomorphic in the unit disk, and let $f$ be a function in the class $S$ for which $\operatorname{Re} L$ achieves its maximum on $S$. Then $L$ is said to be of rational type if the expression $L\left(f^{2} /(f-w)\right)$, which occurs in Schiffer's differential equation, is a rational function of $w$. Various equivalent formulations of "rational type" are found and an application to the process of arc truncation of support points of $S$ is made.
\end{abstract}

1. Introduction. Let $H(\Delta)$ be the topological linear space of holomorphic functions on the unit disk $\Delta=\{z \in \mathbf{C}:|z|<1\}$. The class $S$ is the subset of $H(\Delta)$ consisting of univalent functions $h$ with the normalization $h(0)=0, h^{\prime}(0)=1$. We suppose $L$ is a complex-valued continuous linear functional on $H(\Delta)$, that is $L \in H(\Delta)^{*}$, and that $L$ is nonconstant on $S$. Then any function $f \in S$ for which $\operatorname{Re} L(f)=\max \operatorname{Re}\{L(h): h \in S\}$ must map $\Delta$ onto the complement of an analytic $\operatorname{arc} \Gamma_{f}$ satisfying (except possibly at the finite endpoint)

$$
L\left(f^{2} /(f-w)\right)(d w / w)^{2}>0 \quad\left(w \in \Gamma_{f}\right) .
$$

(See $[\mathbf{8}, \mathbf{7}, \mathbf{4}]$.) Such a function $f$ is called a support point of $S$ (corresponding to $L)$.

In the most important examples - coefficient functionals, or more generally evaluation of a derivative of some order at some point of $\Delta$, or a linear combination of such functionals- $L\left(f^{2} /(f-w)\right)$ is a rational function of $w$, and in a recent paper [5] P. L. Duren, Y. J. Leung, and M. M. Schiffer defined $L$ to be of rational type if $L\left(h^{2} /(h-w)\right)$ is rational for all $h \in S$. This requirement appears to be excessively strong since only support points of $S$, in fact only support points which maximize $\operatorname{Re} L$ necessarily occur in (1). We show, however, in Theorem 1 that if $L\left(h^{2} /(h-w)\right)$ is rational for a single $h \in S$, then it is rational for any $h \in S$, and in fact $L$ is simply a finite linear combination of functionals of the form $h \rightarrow h^{(n)}\left(z_{0}\right)$ ( $n$ a nonnegative integer and $\left.z_{0} \in \Delta\right)$. The key to the proof is the following representation of $L$ :

$$
L(h)=\frac{1}{2 \pi i} \int_{\gamma} h(z) F(z) d z \quad(h \in H(\Delta)),
$$

where $\gamma(t)=r e^{i t}(0 \leq t \leq 2 \pi), 0<r<1, F$ is holomorphic for $|z| \geq r$, and $F(\infty)=0$. The function $F$ is obtained from the well-known Toeplitz representation $[9]$

$$
L(h)=\sum_{0}^{\infty} a_{n} b_{n} \quad\left(h(z)=\sum_{0}^{\infty} a_{n} z^{n}, \varlimsup \lim \left|b_{n}\right|^{1 / n}<1\right)
$$

Received by the editors September 16, 1983.

1980 Mathematics Subject Classification. Primary 30C55, 30C70.

(C) 1984 American Mathematical Society $0002-9939 / 84 \$ 1.00+\$ .25$ per page 
by defining $F(z)=\sum_{0}^{\infty} b_{n} / z^{n+1}$. This function, subject to the conditions stated, is uniquely determined by $L$, but we can of course make use of slight analytic continuations of $F$ and deform the circular contour $\gamma$ into certain other closed curves without changing the value of the integral in (2). Also, we can, if we wish, drop the first two terms $b_{0} / z+b_{1} / z^{2}$ of the Laurent expansion of $F$ about $\infty$ without changing the corresponding support points. In fact, if $J$ is the functional corresponding as in (2) to the function $F(z)-b_{0} / z-b_{1} / z^{2}$, and if $h \in S$, then $L(h)=b_{1}+J(h)$. In addition to the three equivalent formulations of "functional of rational type" already alluded to, Theorem 1 contains the fourth formulation: The function $F$ in (2) is rational. Thus we can restate the definition of "rational type" as follows.

DEFinition. The functional $L \in H(\Delta)^{*}$ is of rational type if any one of the conditions (a), (b), (c), (d) of Theorem 1 holds.

We observe from (2) that for any $f \in S$,

$$
L\left(f^{2} /(f-w)\right)=\frac{1}{2 \pi i} \int_{\gamma} \frac{f(z)^{2}}{f(z)-w} F(z) d z
$$

for $w \in \mathbf{C} \backslash f(\Delta)$, while the last integral furnishes an analytic continuation of $L\left(f^{2} /(f-w)\right)$ to the exterior of the closed Jordan curve $f \circ \gamma$. Our initial lemma provides a new relation between the functions $L\left(f^{2} /(f-w)\right)$ and $F$ as follows. First we note that

$$
f^{2} /(f-w)=f+w+w^{2} /(f-w)
$$

and therefore that

$$
L\left(f^{2} /(f-w)\right)=L(f)+w L(1)+w^{2} L(1 /(f-w)) .
$$

The lemma then shows how $F$ can simply and with great symmetry be expressed in terms of $L(1 /(f-w))$. Later we make use of the fact, exhibited by the last identity, that $L\left(f^{2} /(f-w)\right)$ is rational exactly when $L(1 /(f-w))$ is rational. We remark that the method of the lemma can easily be adapted to express $F(z)-b_{0} / z-b_{1} / z^{2}$ in terms of $L\left(f^{2} /(f-w)\right)$.

Several recent papers on $S([\mathbf{1}, \mathbf{2}, \mathbf{3}, \mathbf{6}])$ have dealt with truncation of the omitted arc $\Gamma_{f}$ of a support point $f$. If after such a truncation the resulting region is contracted so as to be of the form $g(\Delta)$ with $g \in S$, then $g$ is again a support point of $S$. Indeed, if $f$ maximizes $\operatorname{Re} L$ over $S, L \in H(\Delta)^{*}$, a new functional $J$ can rather explicitly be constructed in terms of $L$ and $\Gamma_{f}$ such that $g$ maximizes $\operatorname{Re} J$. (We shall be more explicit in $\S 4$ below.) In Theorem 2 we show that if $L$ is of rational type, then so is $J$. Thus, roughly speaking, functionals of rational type are preserved by arc truncation. In fact, we shall see that in a certain sense the exact form of the functional is preserved (see (6) and (7)).

2. The dual relationship between $L(1 /(f-w))$ and $F(z)$.

LEMma. Let $f \in S, \gamma$ be a positively oriented closed Jordan curve in $\Delta, F$ holomorphic on and outside $\gamma$ with $F(\infty)=0$. Let

$$
G(w)=\frac{1}{2 \pi i} \int_{\gamma} \frac{1}{f(z)-w} F(z) d z \quad(w \text { outside } f \circ \gamma) \text {. }
$$


Then (after a slight analytic continuation of $G$ )

$$
F(z)=\frac{1}{2 \pi i} \int_{f \circ \gamma} \frac{1}{f^{-1}(w)-z} G(w) d w \quad(z \text { outside } \gamma)
$$

PROOF. Since $F$ is holomorphic on and outside $\gamma$ we can replace $\gamma$ by another positively oriented curve $\delta$, slightly inside $\gamma$, and rewrite $G$ (actually analytically continue $G$ ) as follows.

$$
G(w)=\frac{1}{2 \pi i} \int_{\delta} \frac{1}{f(\zeta)-w} F(\varsigma) d \zeta \quad(w \text { outside } f \circ \delta)
$$

Then

$$
\begin{aligned}
\frac{1}{2 \pi i} \int_{f \circ \gamma} \frac{1}{f^{-1}(w)-z} & G(w) d w \\
& =\frac{1}{2 \pi i} \int_{\delta}\left[\frac{1}{2 \pi i} \int_{f \circ \gamma} \frac{1}{f(\zeta)-w} \frac{1}{f^{-1}(w)-z} d w\right] F(\varsigma) d \zeta .
\end{aligned}
$$

Since $z$ lies outside $\gamma, 1 /\left(f^{-1}(w)-z\right)$ is holomorphic inside and on $f \circ \gamma$. Also, for any $\zeta$ on the contour $\delta, f(\zeta)$ is inside $f \circ \gamma$. Hence, by Cauchy's integral formula, the value of the expression in square brackets is

$$
-\left.\frac{1}{f^{-1}(w)-z}\right|_{w=f(\varsigma)}=\frac{1}{z-\varsigma} .
$$

Therefore

$$
\frac{1}{2 \pi i} \int_{f \circ \gamma} \frac{1}{f^{-1}(w)-z} G(w) d w=\frac{1}{2 \pi i} \int_{\delta} \frac{F(\varsigma)}{z-\varsigma} d \varsigma=F(z)
$$

as asserted. We remark that in a similar way (5) implies (4).

\section{The equivalent formulations of "rational type".}

THEOREM 1. Let $L \in H(\Delta)^{*}$ with corresponding function $F$ as in (2). Then the following statements are equivalent:

(a) There exists $f \in S$ such that $L\left(f^{2} /(f-w)\right)$ is a rational function of $w$.

(b) The function $F$ is rational.

(c) $L$ is a finite linear combination of functionals of the form $h \rightarrow h^{(n)}\left(z_{0}\right)$, where $n$ is a nonnegative integer (order of the derivative) and $z_{0} \in \Delta .\left(n\right.$ and $z_{0}$ can vary from term to term.)

(d) The function $L\left(f^{2} /(f-w)\right)$ is rational for every $f \in S$ (Duren, Leung, Schiffer).

Proof. Let $f$ be as in (a). Then as mentioned earlier $L(1 /(f-w))$ is rational or, equivalently, the function $G$ in (4) is rational. Since the poles of $G$ lie inside $f \circ \gamma$, and since $G(\infty)=0, G$ must be a linear combination of functions of the form $\left(w-w_{0}\right)^{-k}$, with $w_{0}$ inside $f \circ \gamma$ and $k$ a positive integer. Therefore it follows from (5) that to prove (b) we need only show that the function

$$
F_{0}(z)=\frac{1}{2 \pi i} \int_{f \circ \gamma} \frac{1}{f^{-1}(w)-z} \frac{1}{\left(w-w_{0}\right)^{k}} d w \quad(z \text { outside } \gamma)
$$


is (the restriction of) a rational function. But for $z$ outside $\gamma$ Cauchy's integral formula for the $(k-1)$ st derivative gives

$$
F_{0}(z)=\frac{1}{(k-1) !}\left(\frac{d}{d w}\right)^{k-1}\left[\frac{1}{f^{-1}(w)-z}\right]_{w=w_{0}} .
$$

The right side of this formula is clearly a rational function of $z$, and so (a) implies (b).

The proof that (b) implies (c) is similar: The function $F(z)$ in (2) is a linear combination of terms $\left(z-z_{0}\right)^{-k}$ with $z_{0}$ inside $\gamma$ and $k \geq 1$. Hence for $h \in H(\Delta)$, $L(h)$ is a linear combination of terms of the form

$$
\frac{1}{2 \pi i} \int_{\delta} \frac{h(z)}{\left(z-z_{0}\right)^{k}} d z=\frac{h^{(k-1)}\left(z_{0}\right)}{(k-1) !} .
$$

To prove (c) implies (d) we need consider only a single functional of the form described in (c). The conclusion in (d) then clearly follows, and the proof of Theorem 1 is complete.

4. Truncation. As an application of Theorem 1 we discuss arc truncation of support points. Let $f$ be a support point of $S, g \in S, r>1$, and $f \prec r g$. Geometrically, a portion of the omitted arc of $f$ is removed and $r g$ maps $\Delta$ onto the complement of the remaining arc. Then it is now well known that $g$ is also a support of $S([\mathbf{1}, \mathbf{2}, \mathbf{3}, \mathbf{6}])$. Briefly, if $f$ maximizes $\operatorname{Re} L\left(L \in H(\Delta)^{*}\right), \varphi$ is defined by $f=r g \circ \varphi=g \circ \varphi / \varphi^{\prime}(0)$, and the functional $J$ is defined by $J(h)=L(h \circ \varphi)$, then $J \in H(\Delta)^{*}$ and $g$ maximizes $\operatorname{Re} J$ over $S$. (One also shows that $L$ nonconstant on $S$ implies $J$ nonconstant on $S$.) We assert that if $L$ is of rational type, then so is $J$. In fact the following more general and, at the same time, more explicit theorem holds.

THEOREM 2. Let $L$ be of rational type, say

$$
L(h)=\sum_{k=1}^{K} \sum_{n=0}^{N_{k}} a_{n k} h^{(n)}\left(z_{k}\right) \quad(h \in H(\Delta)),
$$

where $K \geq 1, N_{K} \geq 0(1 \leq k \leq K), a_{n k} \in \mathbf{C}\left(1 \leq k \leq K, 0 \leq n \leq N_{k}\right)$, and $z_{k} \in \Delta(1 \leq k \leq K)$. Then for any $\varphi \in H(\Delta)$ with $\varphi(\Delta) \subset \Delta$ there exist $b_{n k} \in \mathbf{C}\left(1 \leq k \leq K, 0 \leq n \leq N_{k}\right)$ such that

$$
L(h \circ \varphi)=\sum_{k=1}^{K} \sum_{n=0}^{N_{k}} b_{n k} h^{(n)}\left(\varphi\left(z_{k}\right)\right) \quad(h \in H(\Delta)) .
$$

PROOF. By induction one sees that for $n=1,2,3, \ldots$,

$$
(h \circ \varphi)^{(n)}=\sum_{j=1}^{n}\left(\psi_{j n}\right)\left(h^{(j)} \circ \varphi\right)
$$

where $\psi_{j n}$ is a function obtainable from $\varphi$ by differentiation. (More explicitly:

$$
\psi_{j n}=\sum_{\nu_{1}+\cdots+\nu_{j}=n} I_{\nu_{1}, \ldots, \nu_{j}} \varphi^{\left(\nu_{1}\right)} \cdots \varphi^{\left(\nu_{j}\right)}
$$


for certain positive integers $I_{\nu_{1}, \ldots, \nu_{j}}$.) Taking the trivial case $n=0$ into account we can write

$$
(h \circ \varphi)^{(n)}=\sum_{j=0}^{n}\left(\psi_{j n}\right)\left(h^{(j)} \circ \varphi\right) \quad(n=0,1,2, \ldots),
$$

where $\psi_{0 n}=0$ for $n>0$ and $\psi_{00}=1$. Therefore, by (6),

$$
\begin{aligned}
L(h \circ \varphi) & =\sum_{k=1}^{K} \sum_{n=0}^{N_{k}} a_{n k}\left[\sum_{j=0}^{n} \psi_{j n}\left(z_{k}\right) h^{(j)}\left(\varphi\left(z_{k}\right)\right)\right] \\
& =\sum_{k=1}^{K} \sum_{j=0}^{N_{k}}\left[\sum_{n=j}^{N_{k}} a_{n k} \psi_{j n}\left(z_{k}\right)\right] h^{(j)}\left(\varphi\left(z_{k}\right)\right)
\end{aligned}
$$

as required.

Theorem 2 applies to arc truncation only when $\varphi(0)=0$. Then, in the "truncated functional" (7), all the derivatives $h^{(n)}$ are evaluated at points closer to the origin as compared with corresponding points in (6); that is, $\left|\varphi\left(z_{k}\right)\right| \leq\left|z_{k}\right|$. In particular, if $L$ is a linear combination of Maclaurin coefficients $\left(K=1, z_{1}=0\right)$, then so is $J$. Of course this case is very special and can be seen without the theorem.

Finally, we remark that Cauchy's integral formula provides an answer to the following question: If $L \in H(\Delta)^{*}$ with corresponding function $F$ as in (2), and if $\varphi \in H(\Delta)$ with $\varphi(\Delta) \subset \Delta$, then what function $H$ corresponds to the functional $h \rightarrow L(h \circ \varphi)$ ? The answer is:

$$
H(z)=\frac{1}{2 \pi i} \int_{\gamma} \frac{F(\varsigma)}{z-\varphi(\zeta)} d \varsigma \quad(z \text { in unbounded component of } \mathbf{C} \backslash \varphi \circ \gamma)
$$

The author thanks Stephen Ruscheweyh for helpful conversations including raising the question of whether functionals of rational type are preserved in the process of arc truncation.

\section{REFERENCES}

1. L. Brickman and P. L. Duren, Truncation of support points for univalent functions, Complex Variables Theory Appl. 3 (1984), 71-83.

2. L. Brickman and Y. J. Leung, Exposed points of the set of univalent functions, Bull. London Math. Soc. 16 (1984), 157-159.

3. L. Brickman, Y. J. Leung and D. R. Wilken, On extreme points and support points of the class $S$, Ann. Univ. Mariae Curie-Sklodowska (Krzyz special issue) (to appear).

4. L. Brickman and D. R. Wilken, Support points of the set of univalent functions, Proc. Amer. Math. Soc. 42 (1974), 523-528.

5. P. L. Duren, Y. J. Leung and M. M. Schiffer, Support points with maximum radian angle, Complex Variables Theory Appl. 1 (1983), 263-277.

6. W. E. Kirwan and G. Schober, New inequalities from old ones, Math. Z. 180 (1982), 19-40.

7. A. Pfluger, Lineare Extremalprobleme bei schlichten Funktionen, Ann. Acad. Sci. Fenn. Ser. AI 489 (1971).

8. M. M. Schiffer, A method of variation within the family of simple functions, Proc. London Math. Soc. (2) 44 (1938), 432-449.

9. O. Toeplitz, Die linearen vollkommenen Räume der Funktionentheorie, Comment. Math. Helv. 23 (1949), 222-242.

Department of Mathematics and Statistics, State University of New York at Albany, Albany, New York 12222 